\title{
Timescale and Stability in Adaptive Behaviour
}

\author{
Christopher L. Buckley, Seth Bullock, and Netta Cohen \\ School of Computing, University of Leeds, UK \\ $\{$ christb, seth, netta\}@comp.leeds.ac.uk
}

\begin{abstract}
Recently, in both the neuroscience and adaptive behaviour communities, there has been growing interest in the interplay of multiple timescales within neural systems. In particular, the phenomenon of neuromodulation has received a great deal of interest within neuroscience and a growing amount of attention within adaptive behaviour research. This interest has been driven by hypotheses and evidence that have linked neuromodulatory chemicals to a wide range of important adaptive processes such as regulation, reconfiguration, and plasticity. Here, we first demonstrate that manipulating timescales can qualitatively alter the dynamics of a simple system of coupled model neurons. We go on to explore this effect in larger systems within the framework employed by Gardner, Ashby and May in their seminal studies of stability in complex networks. On the basis of linear stability analysis, we conclude that, despite evidence that timescale is important for stability, the presence of multiple timescales within a single system has, in general, no appreciable effect on the May-Wigner stability/connectance relationship. Finally we address some of the shortcomings of linear stability analysis and conclude that more sophisticated analytical approaches are required in order to explore the impact of multiple timescales on the temporally extended dynamics of adaptive systems.
\end{abstract}

\section{Introduction}

Many of the model systems central to artificial life are explicit networks of simple interacting elements. Cellular automata (CA), artificial neural networks (ANNs) and random Boolean networks (RBNs), for instance, have become key tools in understanding what it is for a system to exhibit complex adaptive behaviour. Such models tend to be the subject of various different kinds of question. For example, the generation of different classes of dynamic behaviour (fixed, cyclic, complex, chaotic) has been of interest to $\mathrm{CA}$ and RBN researchers, e.g., [12 whereas those working with ANNs have been interested in questions of evolvability, problem solving and autonomous agent control, amongst others [3]. Interestingly, in answering these questions, the role of timescale within these systems has often been neglected. CA and RBNs typically comprise elements that share the same timescale (and updated with the same frequency), 4]. Similarly, while some continuous-time recurrent neural networks (CTRNNs) comprise neurons with explicit and varied timescales, this property has not received as much attention as others. For example, Beer [3] presents an extensive examination of 
the dynamics of recurrent CTRNN neurons, but only briefly mentions the impact of their time constants. This tendency to downplay timescale is somewhat surprising, since the natural adaptive systems that inspired these models typically involve processes and mechanisms that operate at multiple timescales. In particular, there is growing recognition that slow chemical processes within neural systems can be key to their ability to exhibit stable, sensitive, reconfigurable adaptive behaviour [5-7].

Here, we adopt an approach to understanding stability in complex networks inspired by classic cybernetics research, and adapt it to explore questions of timescale raised by this current work. First, a brief and selective account of the role of timescales in neural systems is presented, before a simple model exhibiting timescale-sensitive dynamics is detailed. Subsequently, a numerical approach to characterising the influence of timescale on stability is undertaken. The results are discussed and future directions are suggested.

\subsection{Neuromodulation and Multiple Timescales}

Neuromodulation is a term used diversely by neuroscientists to identify nontraditional processes acting alongside conventional neurotransmission. Although the term has been in use for over 20 years, the ubiquity of such processes has only recently been acknowledged. The action of a neuromodulator within the nervous system differs significantly from that idealised within the traditional connectionist paradigm: fast, point-to-point, excitatory/inhibitory [6]. Within neuroscience, there is a large and growing literature that associates slow, diffusive, modulatory, chemical mechanisms with a wide range of important adaptive capacities. Turrigiano [7], for instance, suggests that this type of mechanism is important for efficient lifetime adaptation within vertebrate nervous systems. Neuromodulators have also been implicated in triggering plasticity, regulating activity, governing reconfiguration, etc. 6]. However, conjectures on the role of neuromodulation in adaptation are not solely the province of the neuroscience community. There have also been treatments of this issue within the artificial life and adaptive behaviour communities [8]. For instance, the success of GasNets, a novel class of artificial neural network inspired by neuromodulation research [10], as an evolutionary robotics control architecture has generated a number of interesting theories regarding neuromodulation and adaptive behaviour [1]. GasNets consist of a traditional connectionist network over which the diffusion of neuromodulatory gases is modeled. The underlying network is embedded in a 2D space, where each neuron has the potential to emit gas, which diffuses over the network from a point source, affecting the properties of the gas-sensitive neurons that it comes into contact with. This gas mechanism is inspired by the neuromodulator nitric oxide (NO), which is small enough to pass freely through lipid tissue. The emission of NO is thought to be ubiquitous throughout the nervous system, but in general it is not accounted for in artificial models of neuronal systems.

Although GasNets have only been tested on a small range of tasks to date, the ease with which high-quality solutions can be evolved suggests that the pres- 
ence of idealised neuromodulation may increase the evolvability of this class of control system across a range of real-world problems [12. As yet there is little understanding of why this should be the case. While GasNets have been explored via a series of metrics, the contribution that neuromodulation makes to network evolvability remains unclear [13. Aside from this postulated contribution to GasNet evolvability, the inclusion of idealised neuromodulatory mechanisms in a control system could result in greatly enhanced adaptive properties. However, it is unclear whether these benefits are due to the specifics of the chosen abstraction or more fundamental principles underlying neuromodulation. There is some evidence that it is the combination of fast (neurotransmission) and slow (neuromodulation) processes that may be responsible 14 . Indeed, the slow nature of neuromodulation appears crucial to many of its postulated roles. Whether regulating the gross activity in a neural circuit, or maintaining a neural variable within critical bounds via homeostatic plasticity [7], or switching between different modes of circuit behaviour dynamics (e.g., the switch between swimming and the escape reflex in Tritonia, 15]), neuromodulators are often best considered as slow processes that parameterise a fast sub-system. Understanding how to model this interaction across temporal hierarchies remains an open question.

Of course, the presence of explicitly slow elements or processes is not necessary in order to allow a system to exhibit multiple timescales. The flow of activation through a large recurrent network of fast elements may allow different timescales to arise. For instance, Harvey and Thompson [16] evolved circuitry to discriminate between slow oscillatory inputs where the intrinsic timescale of the components (a few nanoseconds) is five orders of magnitude shorter than the dynamics exhibited by the evolved circuit. Furthermore, in small systems, saddle node or homoclinic bifurcations can give rise to slow dynamics even if the underlying nodes are intrinsically fast [17. For example, in most models of spiking neurons the explicit timescales are fast, usually on the order of $10 \mathrm{~ms}$ or less [6], yet in many cases the dynamics of interest extend well beyond these characteristic timescales. However, given that neural substrates support adaptive behaviour at many different temporal scales and that neuromodulators act on a range of timescales typically slower than that of neurotransmission, it seems intuitive that there may be some value in this explicit combination of multiple timescales.

\section{Stability Criteria for Complex Networks}

In a now classic study, Gardner and Ashby [18] investigated stability criteria for large complex systems in terms of the effect of connectivity on the tendency of a system to exhibit a stable point attractor. The relationship between a network's structure and its stability has been of long standing importance, particularly in the field of ecology [19]. At the time, biologists typically assumed that the stability of an ecosystem would increase with its biodiversity (due to mean field averaging). The same issue has significance for systems ranging from traffic networks to the human brain. In each case, Gardner and Ashby argued, we should 
not necessarily expect to observe stability as systems grow in size. Their numerical results characterised the way in which networks of interacting elements become less stable as their interconnectivity increased. This tendency towards stability was subsequently formalised by May [20], who derived a threshold for stability in terms of the mean-square of the strength of the connections and the degree of interconnectivity. In both these studies, the systems are assumed to comprise elements that share a single intrinsic timescale.

Gardner and Ashby [18] and May [20] considered the stability of a linear system $\mathbf{y}=\left(y_{i}, i=1 \ldots N\right)$, given by

$$
\dot{y}_{i}=-y_{i}+\sum_{j=1}^{N} \omega_{i j} y_{j} \quad \text { in vector form : } \quad \frac{d \mathbf{y}}{d t}=\mathbf{A y}
$$

Here, $\mathbf{A}=\boldsymbol{\Omega}-\mathbf{I}$, where $\boldsymbol{\Omega}=\left(\omega_{i j}\right)$ is a matrix of weighted interaction strengths and $\mathbf{I}$ is simply the identity matrix. Such a system is said to be stable when every eigenvalue of $\mathbf{A}$ has a negative real part 21. Gardner and Ashby [18] employed a numerical method to discover the stability of an ensemble of random networks, varying network size, $N$, and network connectivity, $C$ (the probability that any entry of the weight matrix $\Omega$ is non-zero or, equivalently, the probability that any two elements interact). They were able to demonstrate that stability could be compromised by high connectivity.

To derive a threshold for stability, May [20] used analytical results from the field of random matrix theory [2122]. He drew the entries of $\boldsymbol{\Omega}$ from a statistical distribution with zero mean and a mean-square value, $\alpha$. He then derived a critical threshold above which any network has a high probability of instability. Explicitly, he stated that in the limit of large system size $(N \gg 1)$, a system is almost certainly unstable if $N C \alpha^{2}>1$.

This result, generally referred to as the May-Wigner stability theorem, corresponds well with Gardner and Ashby's original findings and still holds as a very important threshold [23. It has been extended recently to demonstrate that the result stills holds for systems in which connections between elements exhibit time delays [24]. However, as yet, the influence of timescale, as distinct from time delay, has not been explored. Recent work within neuroscience and adaptive behaviour suggests that systems involving processes on multiple timescales readily exhibit important classes of adaptive behaviour. Here we apply the approach introduced by Gardner and Ashby [18] and formalised by May [20] to such systems.

\section{Timescale in a Two-Node System}

The analysis described above assumes linearity, yet it is possible to apply the results to non-linear systems if we restrict our attention to behaviour in the vicinity of a specific equilibrium. In this case, we can consider the local behaviour around this equilibrium and determine the stability of the system under a (vanishingly small) perturbation. This process is known as linear stability analysis. It will tell 
us about the local asymptotic behaviour of a non-linear system around a particular equilibrium but tell us nothing about global stability. For example, while a limit cycle cannot be said to be locally stable, it may be globally stable such that under perturbation the system always settles to the same cyclic trajectory.

May's result has been criticised because it relies on this linearization around equilibrium, which is thought to make it inapplicable where perturbations are large or systems exhibit limit sets of higher dimension than a fixed point. While this issue remains open, recent calculations of global dynamics have obtained the May-Wigner stability thresholds as thresholds for global system stability. These results suggest that the May-Wigner theorem may be more universal than originally expected 23. So, while this technique has restricted application to non-linear systems, it may still has the potential to deliver general insight into the dynamics of complex systems.

We will consider a system of equations used to describe continuous-time recurrent neural networks (CTRNNs). The CTRNN is commonplace throughout neuroscience (as a leaky integrator) and evolutionary robotics [3].

$$
\dot{y}_{i}=-\frac{y_{i}}{\tau_{i}}+\frac{\tanh \left[\sum_{j} \omega_{i j} y_{j}+\theta_{i}\right]}{\tau_{i}}
$$

Here $y_{i}$ represents activation at the $i^{\text {th }}$ neuron; $\omega_{i j}$ is a weight on the connection between neurons $i$ and $j ; \theta_{i}$ is the bias value at the $i^{\text {th }}$ neuron; and $\tau_{i}$ is the time constant of the $i^{\text {th }}$ neuron, which defines the rate of leakage or decay of activation. The equation is forward integrated with a simple Euler step method with time slices of $d t=0.005$. Note that $\tau$ represents the explicit timescale of each of the units and it is this parameter that we will concern ourselves with in this work. In this formulation, the sigmoidal transfer function is a hyperbolic tangent rather than the more familiar exponential sigmoid (see e.g., REF 3]. Note that, here, activation does not represent the membrane potential of a neuron, but rather the firing rate, or mean number of spiking events per unit time, averaged over some appropriate time window. In general we can think of the CTRNN equation as a re-description of the firing rate of a given neuron (or ensemble) averaged over some window, $\tau$.

We will first consider a simple two-node system described by equation (2). To determine the linear stability of this system, we must first calculate the coordinates of its equilibrium point. This is located at the intersection of the system's nullclines, each defined by $\dot{y}_{i}=0$. Second, we must calculate the Jacobian of the system at equilibrium, $J$, given by equation (3), (further details can be found in Refs. [17] and [3.)

$$
J=\left(\begin{array}{ll}
\frac{d \dot{y_{1}}}{d y_{1}} & \frac{d \dot{y_{1}}}{d y_{2}} \\
\frac{d \dot{y_{2}}}{d y_{1}} & \frac{d \dot{y_{2}}}{d y_{2}}
\end{array}\right)_{\overline{y_{1}}, \overline{y_{2}}}
$$

Here, $\overline{y_{1}}$ and $\overline{y_{2}}$ are the equilibrium activation values, and the matrix therefore represents the instantaneous interaction between each element around the equilibrium point, and can be analytically calculated. Under these conditions, this 

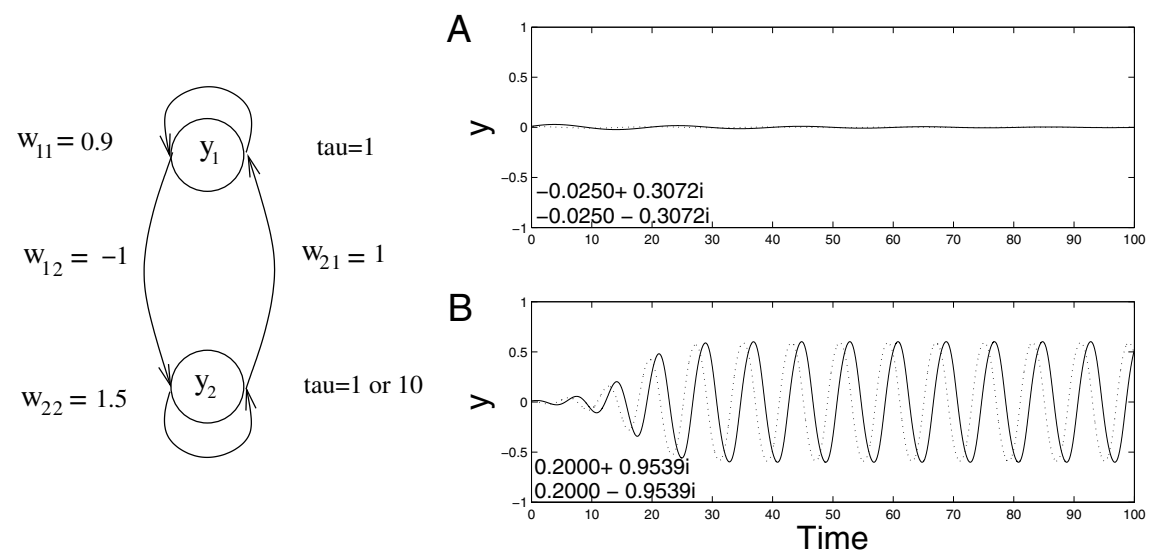

Fig. 1. Variation in the behaviour of a simple two-node circuit with recurrent links (parameterized as shown, left), due to manipulating the timescale of its component elements. In each case, the system is released from an initial condition $\left(y_{1}=y_{2}=0.01\right)$ in the vicinity of the equilibrium at $\overline{y_{1}}=\overline{y_{2}}=0$. A. $\tau_{1}=1, \tau_{2}=10$ : The system exhibits stability. B. $\tau_{1}=\tau_{2}=1$ : the system diverges from equilibrium to a limit cycle. Eigenvalues of the Jacobian for each system are shown alongside the plots.

matrix is equivalent to $\mathbf{A}$ in May's formulation. We can now determine whether the system is stable by requiring that the real parts of each eigenvalue of the matrix are negative.

In Refs. [18] and 20] the timescales of all the elements within a system are assumed to be equal. Here we consider the consequences of relaxing this assumption. In general determining the contents of the Jacobian matrix requires us to calculate complex terms that depend on the first order differential of the CTRNN sigmoidal transfer functions. By stipulating that $\theta_{1}=\theta_{2}=0$ we guarantee that there is a system equilibrium at $\overline{y_{1}}=\overline{y_{2}}=0$, which simplifies the Jacobian, thus:

$$
J=\left(\begin{array}{cc}
\frac{\omega_{11}-1}{\tau_{1}} & \frac{\omega_{12}}{\tau_{1}} \\
\frac{\omega_{21}}{\tau_{2}} & \frac{\omega_{22}-1}{\tau_{2}}
\end{array}\right)
$$

We can rewrite equation (3) in vector form equivalent to equation (1) for a system with multiple timescales as $\mathbf{A}=(\boldsymbol{\Omega}-\mathbf{I}) \tau^{-1}$, where $\tau$ is a vector of the damping times, $\tau_{i}$, for each of element. The question here is what effect this has on the dynamics? To understand this we will consider an example of a coupled two-node system parameterized as illustrated in figure 1.

Figure 1 depicts the behaviour of the coupled system for $\tau_{2}=1$ and $\tau_{2}=10$ (holding $\tau_{1}=1$ constant) from the same initial conditions $\left(y_{1}=0.01, y_{2}=0.01\right.$ ). For $\tau_{2}=10$ the system is locally stable, converging to equilibrium after a small perturbation. In contrast, for $\tau_{2}=1$ the equilibrium at $y_{1}=y_{2}=0$ is unstable. Even though the system is initially perturbed only a small distance from this 
equilibrium, the trajectory diverges to a limit cycle. In fact, as we alter $\tau_{2}$ the system undergoes a subcritical Hopf bifurcation [17]. Is this bifurcation reflected in the linear stability analysis? From equation (4) we can determine that the real parts of each eigenvalue change from positive to negative as we increase $\tau_{2}$ (see figure 1), indicating a transition from local instability to local stability 1

In this simple case, timescale (as well as connectivity and weight strengths) affects system stability. It is interesting to note the direction of this influenceincreasing timescale separation increases system stability. This begs the question: what effect does timescale have on larger systems, and does it interfere with the relationship described by Gardner and Ashby, and formalised by May?

\section{Larger Systems}

In the previous section, we have outlined how linear stability analysis can shed light on the dynamics around an equilibrium position in a non-linear system. For the small system considered above, varying the timescale parameters, $\tau$, brought about a Hopf bifurcation, altering the system's dynamics such that it ceased to exhibit a stable equilibrium. Could timescale have a similar effect on the stability of larger systems? Gardner and Ashby [18] and May [20] considered the effect of both connectivity, $C$, and mean-square weight value, $\alpha$, on stability, but assumed that the damping time of each of the system's elements was unity. In this section we will relax this assumption. To achieve this, we will establish numerically the relationships between probability of stability and both $C$ and $\alpha$ for networks with all $\tau_{i}=1$, and compare this with the same relationships for networks with $\tau_{i}$ uniformly distributed over three orders of magnitude.

The basic form of these relationships, depicted in figure 2 is intuitive. At low $\alpha$ or $C$, networks have a high probability of stability, which decreases as $\alpha$ or $C$ increase. Figure 2]s vertical dotted lines represent the critical threshold derived by May. Predictably, the correspondence between the (asymptotically derived) threshold and the numerical results increases with network size, as does the steepness of the numerically derived "phase transition". However, less predictably, there appears to be little difference between the stability of networks comprising elements with shared, unitary timescale and networks comprising elements with widely varying timescale. In contrast to the example given in section 3 above, multiple timescales have little effect on the stability threshold, or on the general character of the relationship.

Our paired design allows us to confirm that if a network below the MayWigner threshold is stable with unitary timescale elements, the same network will generally be stable if those timescales vary widely. However, for networks above the May-Wigner threshold, in all plots the probability of stability in timescaleseparated networks is slightly, but systematically, lower than the probability of

\footnotetext{
${ }^{1}$ As this analysis only concerns the local behaviour around the equilibrium, it tells us nothing about subsequent trajectories. Nevertheless, the bounded nature of this system and the fact that it can only exhibit one point equilibrium guarantees that, where the original equilibrium is unstable, a cyclic attractor will surround it.
} 

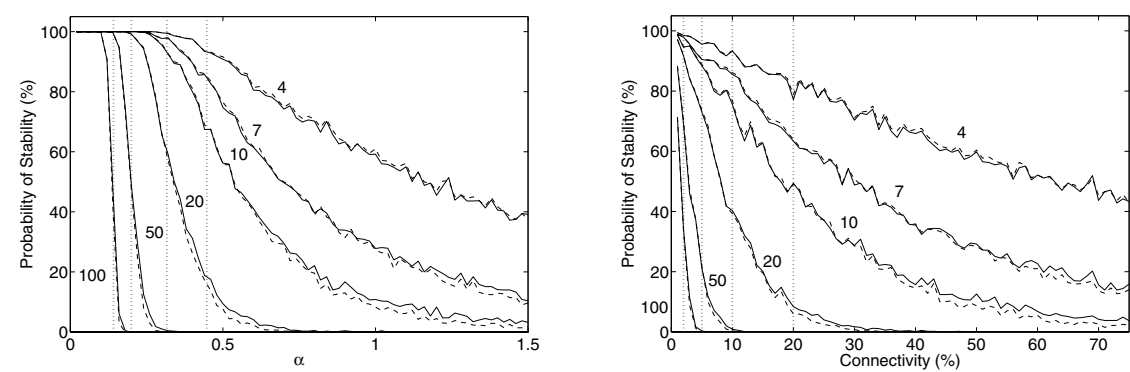

Fig. 2. Probability of stability versus. (a) the root mean square of network weights, $\alpha$, and (b) network connectivity, $C$, for networks of size 4, 7, 10, 20, 50 and 100 nodes (1000 random networks per data point). For (a), $C=50 \%$. For (b), $\alpha=1$. Solid curves depict results for networks with unitary $\tau$ values, dashed curves for the same networks with $\tau$ values uniformly distributed across three orders of magnitude. Vertical lines denote the stability threshold as predicted by the May-Wigner theorem for networks of $100,50,20$ and 10 nodes (reading left to right).

stability in equivalent unitary networks. This may indicate that the presence of multiple timescales encourages the transition to instability. This effect is small, less than $1 \%$ for all network sizes. Although this difference seems negligible in the context of the overall character of the relationship, it would be interesting to investigate its root cause since it is in opposition to the effect of timescale separation demonstrated in section 3 .

So far, we have concerned ourselves only with the real parts of a network's eigenvalues, since these reveal the presence of local stability. While the introduction of multiple timescales has little effect on the probability that these real parts are all negative (indicating local stability), it does have an effect on the imaginary parts of these eigenvalues, which are far more likely to be non-zero in this case. In a simple coupled system, these imaginary parts indicate the manner in which the system transitions to or from equilibrium. If the imaginary parts are zero, the equilibrium is said to be a node, otherwise it is a spiral [17.

The increase in the number of non-zero imaginary eigenvalue parts brought about by the introduction of multiple timescales implies that trajectories around the equilibrium have little or no curvature. We can understand this in terms of the strength of the effects of the different elements that comprise a network. Because each element's entry in the Jacobian matrix (3) is scaled by its inverse timescale, i.e., by $\frac{1}{\tau_{i}}$, slower elements will have a weaker instantaneous influence. Weakening or strengthening an element's influence will not tend to affect local stability, since even a weak effect can displace a system from equilibrium. However, the short-term behaviour of the system will appear to be dominated by fast elements, although slow elements may have a large effect in the long term.

This observation is reminiscent of Ashby's (1960) temporary independence, [25], used to describe how trajectories in the phase space of a complex system may evolve over low-dimensional manifolds if certain variables remain practically 
constant over some period of time. The utility, in this context, of a distinction between interdependence over the short- and long-term is also reminiscent of Simon's 26] attempt to define functional modularity.

\section{Conclusion}

We have demonstrated that, in at least one example, altering the explicit timescale of a network component can effect a transition between stability and instability, despite connectivity and weight parameters remaining fixed. Conversely, we have shown that Gardner and Ashby's stability/connectance relationship and May's critical threshold are largely unaffected by the presence of multiple timescales.

In order to characterise the influence of timescale more satisfactorily, we must move beyond this initial linear stability analysis, and develop tools that allow us to explore the temporally extended non-equilibrium dynamics of systems exhibiting multiple timescales. One potential avenue is the extension of statistical, information-theoretic measures of interdependence, such as mutual information [27, to the task of determining whether sub-systems that are temporally separated might be functionally modular in the sense of Simon [26] or Watson [28]. Such modularity is hinted at by some of the results presented here, and would go a long way toward accounting for the different ways in which neuromodulation has been implicated in underpinning temporally extended adaptive behaviour.

\section{References}

1. Kauffman, S.: The Origins of Order. Oxford University Press, Oxford (1993)

2. Gershenson, C.: Classification of random Boolean networks. In Standish, R.K., Bedau, M.A., Abbass, H.A., eds.: Artificial Life VIII: Proceedings of the Eight International Conference on Artificial Life, MIT Press (2002) 1-8

3. Beer, R.D.: On the dynamics of small continuous-time recurrent neural networks. Adaptive Behavior 3 (1995) 471-511

4. Di Paolo, E.A.: Searching for rhythms in asynchronous Boolean networks. In Bedau, M.A., McCaskill, J.S., Packard, N.H., Rasmussen, S., eds.: Seventh International Conference on Artificial Life, MIT Press, Cambridge, MA (2000)

5. Poggio, T.A., Glaser, D.A., eds.: Exploring Brain Functions: Models in Neuroscience. John Wiley and Sons, New York (1993)

6. Katz, P.S., ed.: Beyond Neurotransmission: Neuromodulation and its Importance for Information Processing. Oxford University Press, Oxford (1999)

7. Turrigiano, G.G.: Homeostatic plasticity in neuronal networks: The more things change, the more they stay the same. Trends in Neuroscience 22 (1999) 221-227

8. Doya, K.: Metalearning and neuromodulation. Neural Networks 15 (2002) 495-506

9. Williams, H.: Homeostatic plasticity in recurrent neural networks. In Schaal, S., Ijspeert, A., Billard, A., Vijayakumar, S., Hallam, J., Meyer, J.A., eds.: Eighth International Conference on the Simulation of Adaptive Behavior, MIT Press, Cambridge, MA (2004) 344-353 
10. . Husbands, P., Philippides, A., Smith, T.M.C., O'Shea, M.: The shifting network: Volume signalling in real and robot nervous systems. In Kelemen, J., Sosik, P., eds.: Sixth European Conference on Artificial Life, Springer, Heidelberg (2001) 23-36

11. Philippides, A.O., Husbands, P., Smith, T.M.C., O'Shea, M.: Fast and loose: Biologically inspired couplings. In Standish, R.K., Bedau, M.A., Abbass, H.A., eds.: Eighth International Conference on Artificial Life, MIT Press, Cambridge, MA (2002) 292-301

12. Smith, T.M.C., Husbands, P., Philippides, A.O., O'Shea, M.: Neuronal plasticity and temporal adaptivity: GasNet robot control networks. Adaptive Behavior 10 (2002) 161-184

13. Smith, T.M.C., Husbands, P., O'Shea, M.: Not measuring evolvability: Initial exploration of an evolutionary robotics search space. In: Congress on Evolutionary Computation. IEEE Press (2001) 9-16

14. Buckley, C., Bullock, S., Cohen, N.: Toward a dynamical systems analysis of neuromodulation. In Schaal, S., Ijspeert, A.J., Vijayakumar, A.B.S., Hallam, J., Meyer, J.A., eds.: Eighth International Conference on Simulation of Adaptive Behavior, MIT Press, Cambridge, MA (2004) 334-343

15. Hooper, S.L.: Neural circuits: Functional reconfiguration. In: Nature Encyclopedia of Life Science, Nature Publishing Group, London (2001)

16. Harvey, I., Thompson, A.: Through the labyrinth evolution finds a way: A silicon ridge. In Higuchi, T., Iwata, M., Weixin, L., eds.: First International Conference on Evolvable Systems, Springer-Verlag, Heidelberg (1997) 406-422

17. Strogatz, S.H.: Nonlinear Dynamics \& Chaos. Addison-Wesley, Reading MA (1994)

18. Gardner, M.R., Ashby, W.R.: Connectance of large dynamic (cybernetic) systems: Critical values for stability. Nature 228 (1970) 784-784

19. McCann, K.S.: The diversity-stability debate. Nature 405 (2000) 228-233

20. May, R.M.: Will a large complex system be stable. Nature 238 (1972) 413-414

21. Mehta, M.L.: Random Matrices. Academic Press, New York (1967)

22. Wigner, E.P.: Gruppentheorie und Ihre Anwendung auf die Quantenmechanik der Atomspektren, trans. J. J. Griffin. Academic Press, New York (1959)

23. Sinha, S., Sinha, S.: Evidence of universality for the May-Wigner stability theorem for random networks with local dynamics. Phy. Rev. Let. E 71 (2005) 1-4

24. Jirsa, V.K., Ding, M.: Will a large complex system with time delays be stable. Physical Review Letters 93 (2004) 070602

25. Ashby, W.R.: Design for a Brain. Chapman and Hall, London (1960)

26. Simon, H.A.: The Sciences of the Artificial. MIT Press, Cambridge, MA (1969)

27. Tononi, G., Edelman, G., Sporns, O.: Complexity and coherency: integrating information in the brain. Trends in Cognitive Sciences 2 (1998) 474-483

28. Watson, R.A.: Modular interdependency in complex dynamical systems. In Bilotta, E., ed.: Workshop Proceedings of Alife VIII, MIT Press, Cambridge, MA (2003) 